Supporting Information

\title{
Adsorption/Desorption and Transport of Water in Two-Dimensional Hexagonal Mesoporous Silica
}

Hideki Yanagihara, ${ }^{\dagger}$ Kyohei Yamashita, ${ }^{\dagger}$ Akira Endo, ${ }^{+}$and Hirofumi Daiguji ${ }^{*}{ }^{\dagger}$

${ }^{\dagger}$ Division of Environmental Studies, Graduate School of Frontier Sciences, The University of Tokyo, 5-1-5 Kashiwanoha, Kashiwa 277-8563, Japan

National Institute of Advanced Industrial Science and Technology (AIST), AIST Tsukuba Central 5-2, 1-1-1 Higashi, Tsukuba, Ibaraki 305-8565, Japan

*To whom correspondence should be addressed.

E-mail: daiguji@k.u-tokyo.ac.jp, Phone: +81 47136 4658, Fax: +81 471364659 
S1. Comparison of adsorption-desorption isotherms of water on Zr-MPS obtained by gravimetric and volumetric methods.

Figure S1 shows the adsorption-desorption isotherms of water on Zr-MPS obtained by gravimetric and volumetric methods at $298 \mathrm{~K}$.

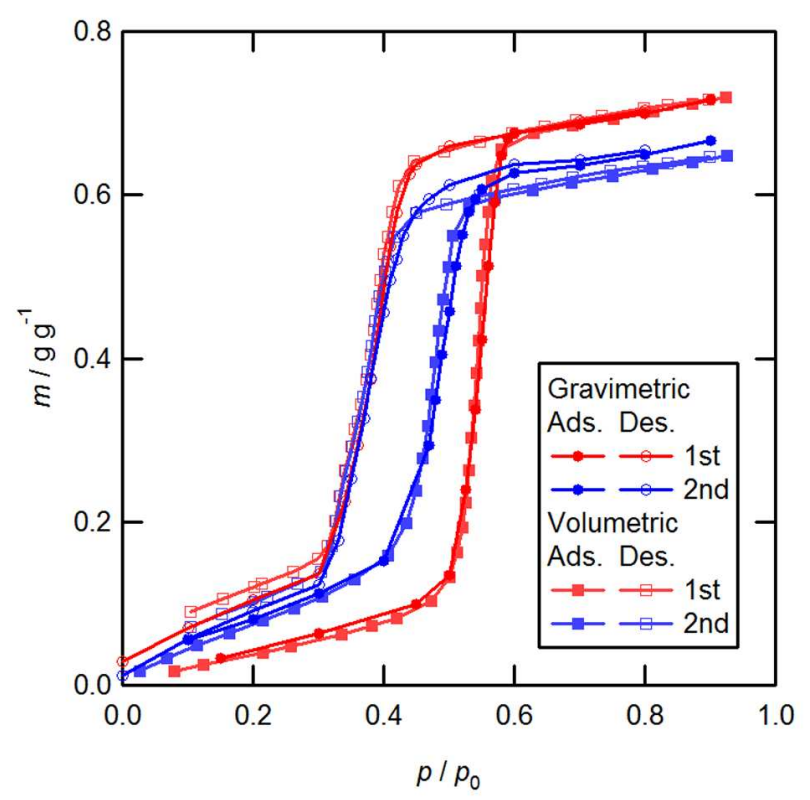

Figure S1. Adsorption-desorption isotherms of water on Zr-MPS obtained by gravimetric and volumetric methods at $298 \mathrm{~K}$. 
S2. Enlarged view of Figure 5 (Curve fitting of relaxation curves)

Figures S2a-I, S2a-II, S2b-I, and S2b-II show the enlarged view around $t=0$ of Figures 5a-I, 5a-II, 5b-I, and 5b-II, respectively.
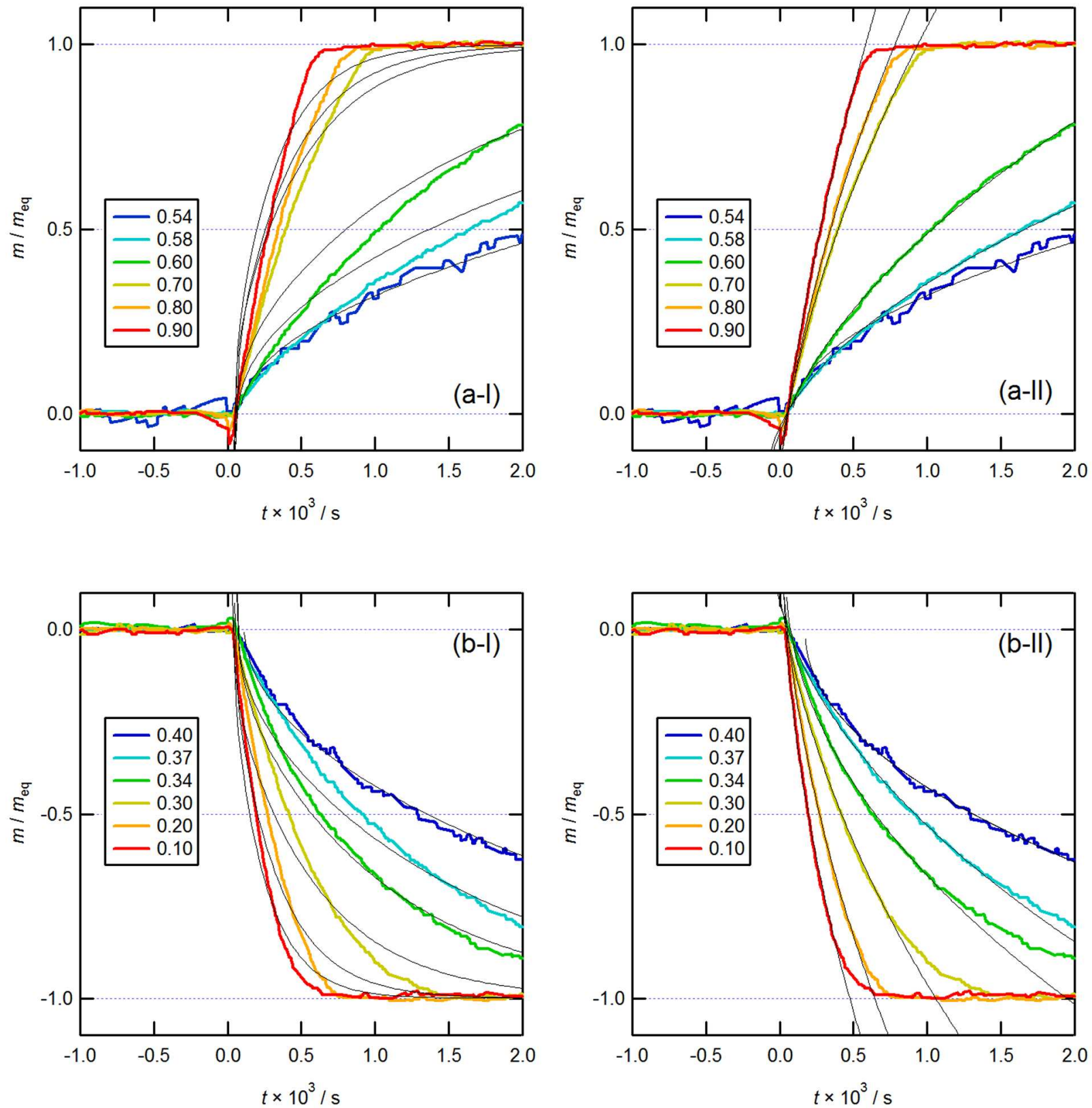
Figure S2. Curve fitting of the relaxation curves of (a) water adsorption and (b) water desorption shown in Figure 4 . The adsorbed mass, $m$, was normalized by the equilibrium value, $m_{\text {eq. }}$ The fitting functions are (I) $m / m_{\text {eq }}=\operatorname{sgn}\left(m / m_{\text {eq }}\right)\left[1-\sum_{n=1}^{10}\left(C_{n} \sin \zeta_{n} / \zeta_{n}\right) \exp \left(-\zeta_{n}^{2} k_{1} t\right)\right]$ (eq. 2) and (II) $m / m_{\text {eq }}=\operatorname{sgn}\left(m / m_{\text {eq }}\right)\left(k_{2} t\right)^{1 / 2}-A$ (eq. 3). 
S3. Exact solution of water diffusion in mesoporous silica in continuum dynamics

Consider one-dimensional water diffusion in a parallel plate of thickness $2 d$. Non-dimensional water concentration in mesoporous silica, distance from the interface, and time are defined as $c^{\prime}=\left(c-c_{0}\right) /\left(c_{\mathrm{eq}}-c_{0}\right), x^{\prime}=x / d$, and $t^{\prime}=(D t) / d^{2}$, respectively. Here, $c_{0}$ and $c_{\mathrm{eq}}$ are the initial and equilibrium water concentrations and $D$ is diffusivity of water in mesoporous silica. The interfaces are at $x^{\prime}= \pm 1$, and the water concentration distribution at any instant must be symmetrical about the midplane $\left(x^{\prime}=0\right)$. If the adsorbed water is initially at a uniform concentration $\left(c^{\prime}\left(x^{\prime}, 0\right)=0\right)$ and comes into contact with air with different humidity at the interface $\left(c^{\prime}\left( \pm 1, t^{\prime}\right)=1\right)$, the resulting concentration profiles can be obtained by solving the following equation:

$$
\frac{\partial c^{\prime}}{\partial t^{\prime}}=\frac{\partial^{2} c^{\prime}}{\partial x^{\prime 2}}
$$

An exact solution to this equation is of the form

$$
c^{\prime}=1-\sum_{n=1}^{\infty} C_{n} \exp \left(-\zeta_{n}^{2} t^{\prime}\right) \cos \left(\zeta_{n}\left(1-x^{\prime}\right)\right)
$$

where $\zeta_{n}=(\pi / 2)(2 n-1)$ and $C_{n}=\left(4 \sin \zeta_{n}\right) /\left(2 \zeta_{n}+\sin \left(2 \zeta_{n}\right)\right)$. The amount of adsorbed water as a function of time can be given by 


$$
m^{\prime}=\int_{0}^{1} c^{\prime} d x^{\prime}=1-\sum_{n=1}^{\infty} \frac{C_{n} \sin \zeta_{n}}{\zeta_{n}} \exp \left(-\zeta_{n}^{2} t^{\prime}\right)
$$

Here, it was assumed that $m^{\prime}=0$ at $t^{\prime}=0$ at the initial condition. For long-time approximation $\left(t^{\prime}>0.2\right)$, the infinite series solution of eq. S3 can be approximated by the first term of the series. Specifically,

$$
m^{\prime}=1-\frac{C_{1} \sin \zeta_{1}}{\zeta_{1}} \exp \left(-\zeta_{1}^{2} t^{\prime}\right)=1-\frac{8}{\pi^{2}} \exp \left(-\frac{\pi^{2}}{4} t^{\prime}\right)
$$

On the other hand, the short-time approximation $\left(t^{\prime}<0.2\right)$ can be given by

$$
m^{\prime}=\left(\frac{4}{\pi} t^{\prime}\right)^{1 / 2}
$$

Figure S3 shows the $m^{\prime}-t^{\prime}$ curves obtained from eqs. S3-S5. Figure S3 shows that eq. S3 is well approximated by eq. S4 at $t^{\prime}>0.2$ (or $0.5<m^{\prime}<1$ ), whereas it is approximated by eq. S5 at $t^{\prime}<0.2$ (or $0<m^{\prime}<0.5$ ). Eqs. S3-S5 can also be given in the dimensional form:

$$
\begin{aligned}
& \frac{m}{m_{\mathrm{eq}}}=1-\sum_{n=1}^{\infty} \frac{C_{n} \sin \zeta_{n}}{\zeta_{n}} \exp \left(-\zeta_{n}^{2} \frac{D}{d^{2}} t\right), \\
& \frac{m}{m_{\mathrm{eq}}}=1-\frac{8}{\pi^{2}} \exp \left(-\frac{\pi^{2}}{4} \frac{D}{d^{2}} t\right),
\end{aligned}
$$




$$
\frac{m}{m_{\mathrm{eq}}}=\left(\frac{4}{\pi} \frac{D}{d^{2}} t\right)^{1 / 2}
$$

where $m_{\mathrm{eq}}$ is the equilibrium adsorbed mass of water. In Figure 5, the time course of $\mathrm{m} / \mathrm{m}_{\mathrm{eq}}$ is fitted by the following two equations: $m / m_{\mathrm{eq}}=1-\sum_{n=1}^{10}\left(C_{n} \sin \zeta_{n} / \zeta_{n}\right) \exp \left(-\zeta_{n}^{2} k_{1} t\right)$ (eq. 2) and $m / m_{\text {eq }}=\left(k_{2} t\right)^{1 / 2}-A$ (eq. 3 ), where $k_{1}, k_{2}$, and $A$ are fitting parameters. If eq. 3 is the short-time approximation of eq. $2, k_{2}=(4 / \pi) k_{1}$ and $A=0$.

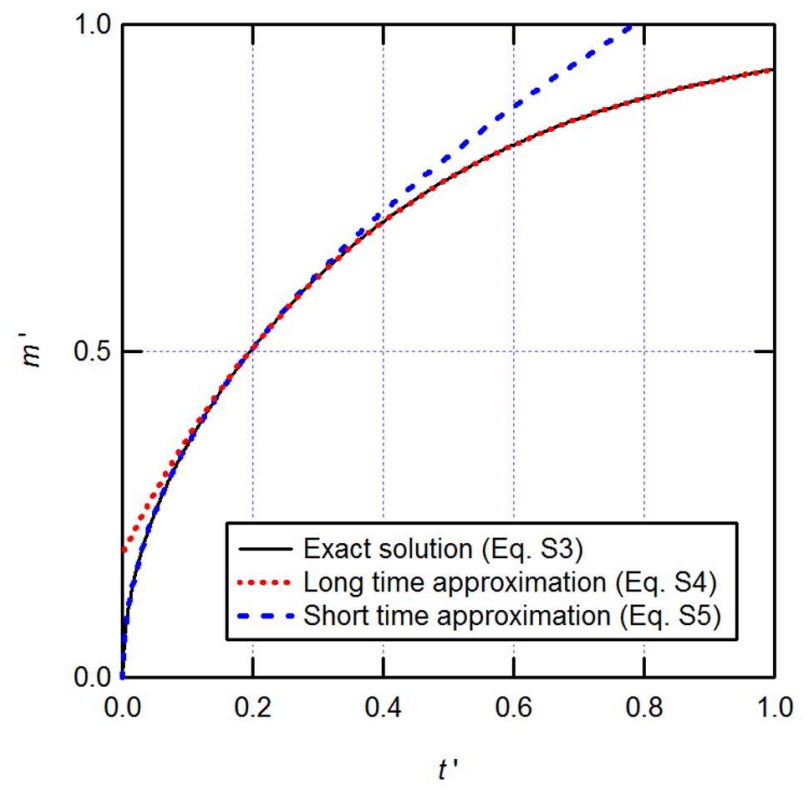

Figure S3. $m^{\prime}-t^{\prime}$ curves obtained from eqs. S3-S5. 